\title{
Utility of Non-EPI DWI MRI in the Management of Pediatric Cholesteatoma
}

\author{
${ }^{1}$ Department of ENT, Columbia Asia Referral Hospital, \\ Yeshwanthpur, Karnataka, India \\ 2Department of Radiology, Columbia Asia Referral Hospital, \\ Yeshwanthpur, Karnataka, India \\ ${ }^{3}$ Department of Otorhinolaryngology, Columbia Asia Referral \\ Hospital, Yeshwanthpur, Karnataka, India
}

Sreenivasa Murthy T.M. ${ }^{1}$ Anita Nagadi ${ }^{2}$ Sneha Krishna Mohan ${ }^{3}$

\begin{abstract}
Address for correspondence Sreenivasa Murthy TM, MBBS, PG Diploma, DNB, Department of ENT, Columbia Asia Referral Hospital, Yeshwanthpur 560055, Karnataka, India (e-mail: tmsreemurthy@gmail.com).
\end{abstract}

\begin{abstract}
Introduction Cholesteatoma in children can be acquired or congenital and is generally extensive. The most commonly used technique for the removal of cholesteatoma is intact canal wall mastoidectomy. This invariably leads to second-look surgery to assess for recurrence or residual cholesteatoma. Magnetic resonance imaging (MRI) with non-echo-planar imaging (non-EPI) diffusion-weighted imaging (DWI) is an accurate noninvasive imaging option that can be used in diagnosing primary cholesteatoma. This can also be used to diagnose residual or recurrent cholesteatoma in patients who have undergone intact canal wall mastoidectomy.

Case Report A 7-year-old male patient presented with a 1-week history of foulsmelling discharge from the right ear. On examination, a polypoidal mass with keratinized debris was seen. A diagnosis of cholesteatoma of right ear was made. Imaging with high-resolution computed tomography (HRCT) of the temporal bone and non-EPI DWI MRI confirmed the same. The child underwent an intact canal wall

Keywords

- non-EPI DWI MRI

- high-resolution computed tomography

- relook surgery mastoidectomy and was followed up at 2 years with non-EPI DWI MRI to monitor any residual or recurrent cholesteatoma.

Conclusion Non-EPI DWI MRI provides a convenient, accurate, and noninvasive method of evaluating and monitoring for residual or recurrent cholesteatoma and thus helps avoid unnecessary second-look surgery.
\end{abstract}

\section{Introduction}

Cholesteatoma in children can be congenital or acquired. ${ }^{1}$ It is generally extensive because of the anatomy of mastoid that is usually diploic or cellular., ${ }^{2,3}$ The traditional treatment of cholesteatoma is a canal wall down (CWD) mastoidectomy that results in a large cavity with its associated problems. To circumvent the problems of cavity, an alternative option is intact canal wall (ICW) mastoidectomy. The disadvantage of ICW technique is the possibility of recurrence or residual cholesteatoma that further requires a relook surgery at regular intervals. ${ }^{4.5}$ Imaging with high-resolution computed tomography (HRCT) as a noninvasive modality offers limited information about specificity regarding cholesteatoma in the postoperative setting. ${ }^{6}$ Magnetic resonance imaging (MRI) with non-echo-planar imaging (non-EPI) diffusion-weighted imaging (DWI) is a noninvasive option for diagnosing and predicting the extent of cholesteatoma as well as monitoring postoperative residual or recurrent cholesteatoma.?

We present a case of a 7-year-old child with extensive cholesteatoma managed with ICW technique and monitored using this non-EPI DWI MRI.

\section{Case Report}

A 7-year-old male patient presented with history of foul-smelling discharge from the right ear. On examination under microscope, he was found to have a polypoidal mass in

\section{received}

October 5, 2018

accepted

December 4, 2018
DOI https://doi.org/

10.1055/s-0039-1678467

ISSN 2581-9607
Copyright @2019 Indian Society of Otology
License terms

(요 (1) $\Theta \circledast$ 
the right external auditory canal, which after excision showed a retraction pocket in the attic area with keratinizing debris. The left ear was normal. A diagnosis of right ear chronic suppurative otitis media with suspicion of cholesteatoma was made. HRCT of the temporal bone revealed opacification of the right middle ear cavity, predominantly in the epitympanum, contiguously extending through aditus ad antrum into the mastoid with complete opacification of mastoid air cells. There was truncation of the scutum and widening of Prussak's space. Erosion of the body, short and long process of the incus with mild truncation of the head of the malleus was seen. The stapes was normal (-Fig. 1). A non-EPI DWI MRI revealed a $5 \times 6 \times 7 \mathrm{~mm}(\mathrm{AP} \times \mathrm{T} \times \mathrm{CC})$ focus of diffusion restriction in the middle ear and another contiguous $9 \times 6 \times 10 \mathrm{~mm}(\mathrm{AP} \times \mathrm{T} \times \mathrm{CC})$ focus of diffusion restriction in the mastoid (-Fig. 2). Appearances were consistent with a large cholesteatoma with ossicular chain erosion. Pure tone audiometry (PTA) showed minimal conductive hearing loss in the right ear (mean PTA: $-23.30 \mathrm{dBHL}$ ). The patient was offered the options of CWD mastoidectomy or ICW mastoidectomy and tympanoplasty, followed by frequent follow-up visits and MRI screening for residual or recurrent cholesteatoma. The parents opted for an ICW mastoidectomy, and the patient underwent the procedure. Intraoperative findings showed cholesteatoma in the attic that was found filling the posterior two-thirds of mesotympanum and facial recess with no extension into the anterior epitympanum. The incus was absent, malleus was partially eroded, and stapes was found intact. Intraoperative findings correlated with the non-EPI DWI MRI findings. The immediate postoperative period was uneventful. Postoperatively, the patient was followed up clinically after 1 month. Examination under the microscope showed an intact neotympanum and no evidence of cholesteatoma, and postoperative audiogram showed
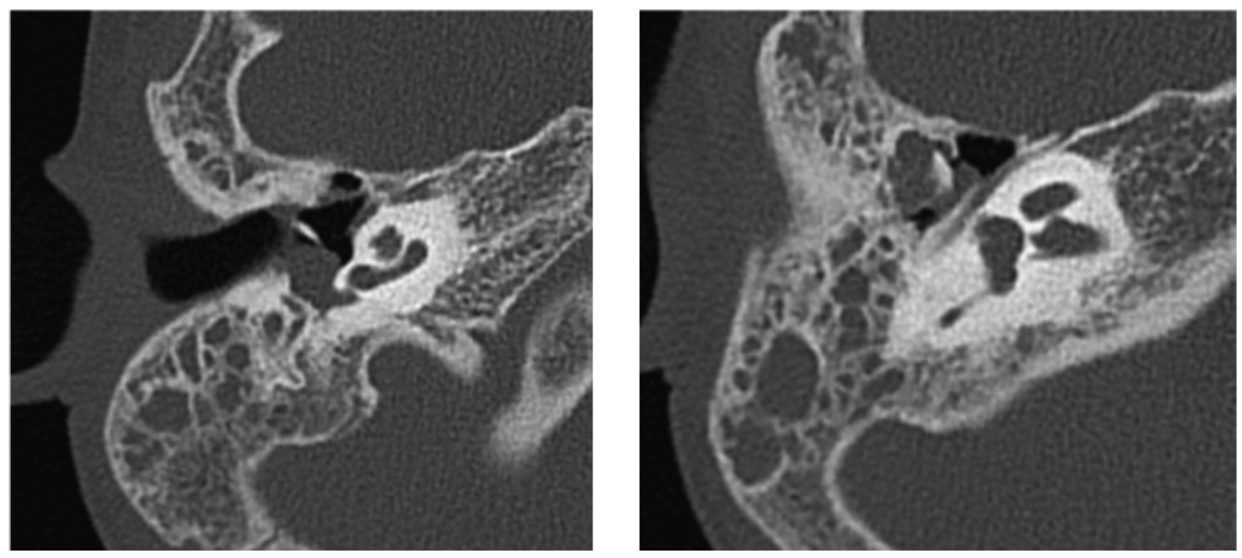

Fig. 1 Axial HRCT of the temporal bones demonstrating opacification of the right mesotympanum and epitympanum (white arrows), with absent long process of the incus and erosions in the body and short process of incus (black arrow). HRCT, high-resolution computed tomography.
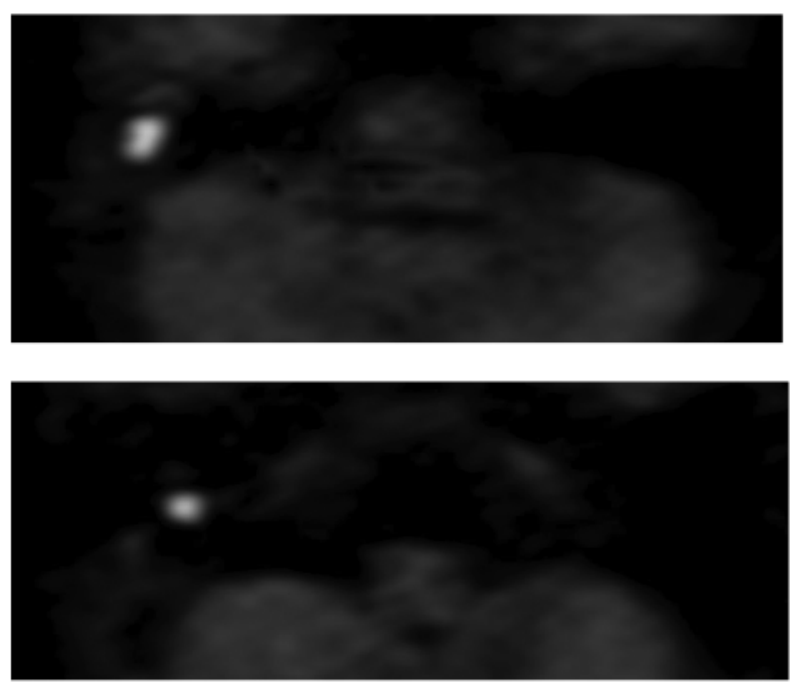

Fig. 2 Axial non-EPI DWI (T2 HASTE) demonstrating large areas of restricted diffusion, seen as bright foci in the right middle ear and mastoid (white arrows) compatible with cholesteatoma. EPI DWI, echo-planar imaging diffusion-weighted imaging. HASTE, half-Fourier acquisition single-shot turbo. mean AC threshold at $36.60 \mathrm{dBHL}$ in the right ear. The patient was followed up at 2 years with HRCT and non-EPI DWI MRI for any evidence of residual or recurrent cholesteatoma. The studies demonstrated the right canal wall up mastoidectomy cavity with no evidence of restricted diffusion to suggest residual or recurrent cholesteatoma (-Figs. 3, 4). The patient is asymptomatic and continues on clinical follow-up.

\section{Discussion and Conclusion}

Cholesteatoma in the pediatric population is aggressive and requires early management to avoid complications. Diagnosis was based on clinical examination and HRCT of the temporal bones to determine extent of the cholesteatoma. Once diagnosed, cholesteatoma removal was done by either the more conventional CWD procedure or the more recent ICW mastoidectomy. Because the conventional CWD mastoidectomy involved extensive dissection and postoperative cavity issues, there is a paradigm shift toward preserving the posterior canal wall and offering ICW mastoidectomy for patients with cholesteatoma. Literature review demonstrates 

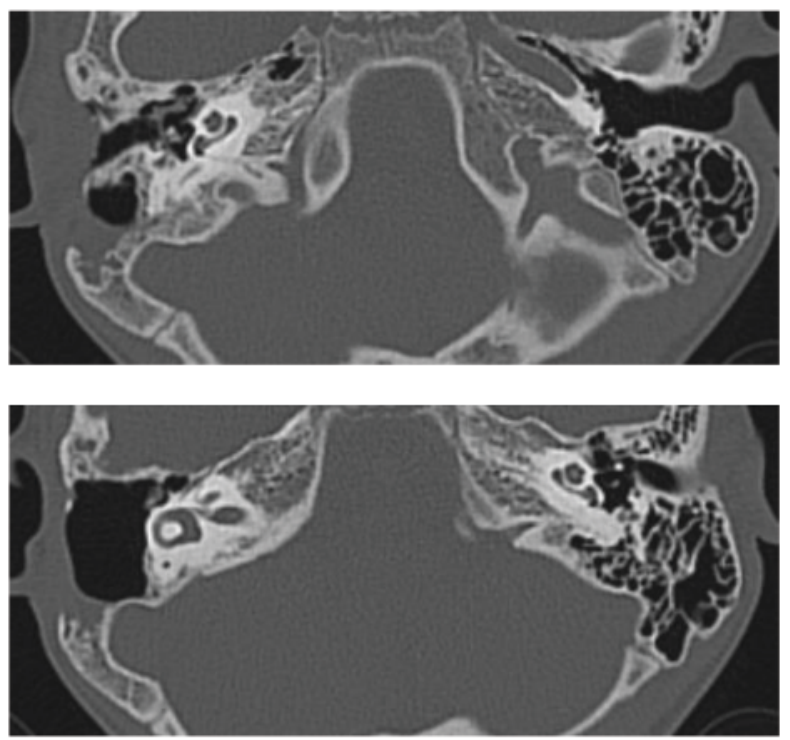

Fig. 3 Follow-up HRCT at 2 years demonstrating a right intact canal wall mastoidectomy with minor right middle ear linear opacities but no mastoid cavity opacification. HRCT, high-resolution computed tomography.
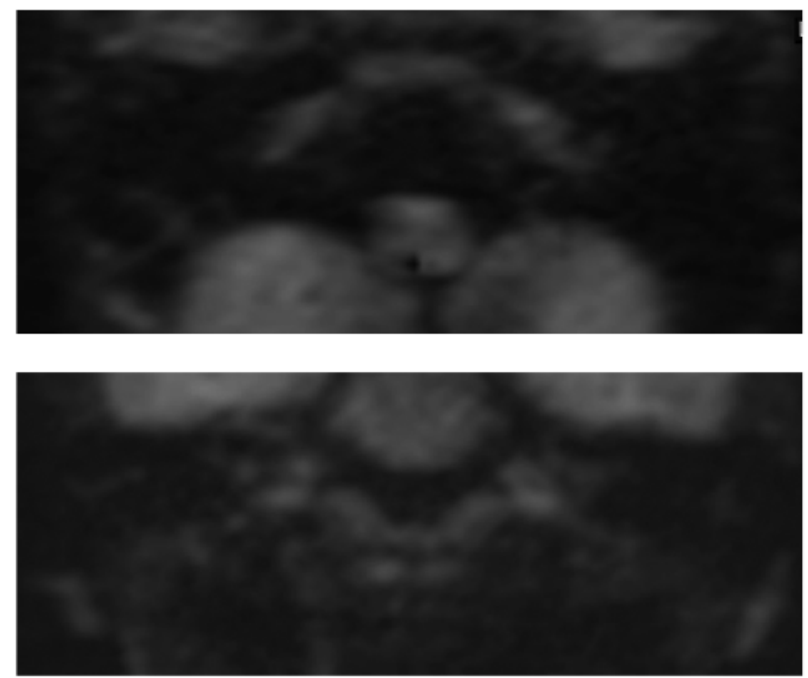

Fig. 4 Axial and coronal non-EPI DWI (T2 HASTE) at the 2-year follow-up demonstrating no foci of restricted diffusion in the right middle ear and mastoid. EPI DWI, echo-planar imaging diffusion-weighted imaging. a very high rate of recurrence or recidivism. The incidence of recurrence and residual cholesteatoma was comparatively higher among patients who underwent ICW mastoidectomy compared with those who underwent CWD mastoidectomy. ICW mastoidectomy requires regular follow-ups and screening for such a recurrence. Traditionally, second-look revision surgery was the most common technique for assessment of recurrence. This meant a mandatory second surgery, and often it was found that the revision surgery was unnecessary as no disease was found. Non-EPI DWI MRI provided a very convenient, less invasive, and an accurate method to evaluate such a recurrence, thereby circumventing the need for invasive methods.

\section{Conflict of Interest}

None declared.

\section{References}

1 Nevoux J, Lenoir M, Roger G, Denoyelle F, Ducou Le Pointe H, Garabédian E-N. Childhood cholesteatoma. Eur Ann Otorhinolaryngol Head Neck Dis 2010;127(4):143-150

2 Glynn F, Osman L, Colreavy M, Rowley H, Dwyer TP, Blayney A. Acute mastoiditis in children: presentation and long term consequences. J Laryngol Otol 2008;122(3):233-237

3 Dornelles C, Costa SS, Meurer L, Schweiger C. Some considerations about acquired adult and pediatric cholesteatomas. Rev Bras Otorrinolaringol (Engl Ed) 2005;71(4):536-545

4 Prasad SC, La Melia C, Medina M, et al. Long-term surgical and functional outcomes of the intact canal wall technique for middle ear cholesteatoma in the paediatric population. Acta Otorhinolaryngol Ital 2014;34(5):354-361

5 Wilson KF, Hoggan RN, Shelton C. Tympanoplasty with intact canal wall mastoidectomy for cholesteatoma: long-term surgical outcomes. Otolaryngol Head Neck Surg 2013;149(2):292-295

6 Estelle's FM, Fernández MM, Bisquert BC, De Castro F, Román IP, Pérez, CM. Contemporary non-echo-planar diffusion-weighted imaging of middle ear cholesteatomas. Radiographic 2012;32:1197-1213

7 Alzahrani M, Alhazmi R, Bélair M, Saliba I. Postoperative diffusion weighted MRI and preoperative CT scan fusion for residual cholesteatoma localization. Int J Pediatr Otorhinolaryngol 2016;90:259-263 